\title{
IdentPMP: identification of moonlighting proteins in plants using sequence-based learning models
}

\author{
Xinyi Liu ${ }^{\text {Equal first author, } 1}$, Yueyue Shen ${ }^{\text {Equal first author, } 1}{ }^{,}$Youhua Zhang ${ }^{1}$, Fei Liu ${ }^{1}$, Zhiyu Ma ${ }^{1}$, Zhenyu Yue $^{\text {Corresp., } 1}$, Yi Yue ${ }^{\text {Corresp }}$ \\ ${ }^{1}$ School of Information and Computer, Anhui Provincial Engineering Laboratory for Beidou Precision Agriculture Information, Anhui Agricultural University, \\ Hefei, Anhui, China \\ Corresponding Authors: Zhenyu Yue, Yi Yue \\ Email address: zhenyuyue@ahau.edu.cn, yyyue@ahau.edu.cn
}

Background. Moonlighting protein refers to a protein that can perform two or more functions. Since the current moonlighting protein prediction tools mainly focus on the proteins in animals and microorganisms , and there are differences in the cells and proteins between animals and plants, these may cause the existing tools to predict plant moonlighting proteins inaccurately. Hence, the availability of a benchmark data set and a prediction tool specific for plant moonlighting protein are necessary.

Methods. This study used some protein feature classes from the data set constructed in house to develop a web-based prediction tool. In the beginning, we built a data set about plant protein and reduced redundant sequences. Then performed feature selection, feature normalization and feature dimensionality reduction on the training data. Next, machine learning methods for preliminary modeling were used to select feature classes that performed best in plant moonlighting protein prediction. And this selected feature was incorporated into the final plant protein prediction tool. After that, we compared five machine learning methods and used grid searching to optimize parameters, and the most suitable method was chosen as the final model.

Results. The prediction results indicated that the eXtreme Gradient Boosting (XGBoost) performed best, which was used as the algorithm to construct the prediction tool, called IdentPMP (Identification of Plant Moonlighting Proteins). The results of the independent test set shows that the area under the precisionrecall curve (AUPRC) and the area under the receiver operating characteristic curve (AUC) of IdentPMP is 0.43 and 0.68 , which are $19.44 \%$ ( 0.43 vs. 0.36$)$ and $13.33 \%$ (0.68 vs. 0.60$)$ higher than state-of-the-art non-plant specific methods, respectively. This further demonstrated that a benchmark data set and a plant-specific prediction tool was required for plant moonlighting protein studies. Finally, we implemented the tool into a web version, and users can use it freely through the URL: http://identpmp.aielab.net/. 
1 IdentPMP: identification of moonlighting proteins in 2 plants using sequence-based learning models

3

4 Xinyi Liü, Yueyue Shen", Youhua Zhang, Fei Liu, Zhiyu Ma, Zhenyu Yue* and Yi Yue* 5

School of Information and Computer, Anhui Provincial Engineering Laboratory for Beidou Precision Agriculture Information, Anhui Agricultural University, Hefei, Anhui Province, China.

\#These authors contributed equally to this work.

Corresponding Author:

Zhenyu Yue (zhenyuyue@ahau.edu.cn) or Yi Yue (yyyue@ahau.edu.cn)

\section{Abstract}

Background. Moonlighting protein refers to a protein that can perform two or more functions. Since the current moonlighting protein prediction tools mainly focus on the proteins in animals and microorganisms, and there are differences in the cells and proteins between animals and plants, these may cause the existing tools to predict plant moonlighting proteins inaccurately. Hence, the availability of a benchmark data set and a prediction tool specific for plant moonlighting protein are necessary.

Methods. This study used some protein feature classes from the data set constructed in house to develop a web-based prediction tool. In the beginning, we built a data set about plant protein and reduced redundant sequences. Then performed feature selection, feature normalization and feature dimensionality reduction on the training data. Next, machine learning methods for preliminary modeling were used to select feature classes that performed best in plant moonlighting protein prediction. And this selected feature was incorporated into the final plant protein prediction tool. After that, we compared five machine learning methods and used grid searching to optimize parameters, and the most suitable method was chosen as the final model.

Results. The prediction results indicated that the eXtreme Gradient Boosting (XGBoost) performed best, which was used as the algorithm to construct the prediction tool, called IdentPMP (Identification of Plant Moonlighting Proteins). The results of the independent test set shows that the area under the precision-recall curve (AUPRC) and the area under the receiver operating characteristic curve (AUC) of IdentPMP is 0.43 and 0.68 , which are $19.44 \%$ ( 0.43 vs. 0.36 ) and $13.33 \%$ (0.68 vs. 0.60$)$ higher than state-of-the-art non-plant specific methods, respectively. This further demonstrated that a benchmark data set and a plant-specific prediction 
36

37

38

39

40

41

42

43

44

45

46

47

48

49

50

51

52

53

54

55

56

57

58

59

60

61

62

63

64

65

66

67

68

69

70

71

72

tool was required for plant moonlighting protein studies. Finally, we implemented the tool into a web version, and users can use it freely through the URL: http://identpmp.aielab.net/.

\section{Introduction}

The continuous accumulation of technology and bioinformatics data in the post-genome era has brought new opportunities and challenges to bioinformatics research. The phenomenon of protein multi functionality in proteomics has also attracted high attention. As more and more proteins are studied in-depth, they are found to have two or more different functions. The idea of one-to-one correspondence between protein and function has been gradually overturned by moonlighting proteins that can perform two or more functions, that usually refers to a single polypeptide chain rather than the product of gene fusion mutation (Jeffery, 1999; Bo et al., 2019). Moonlighting proteins can perform multiple functions simultaneously or alternately due to triggering certain factors. It has been suggested that moonlighting proteins have a combined effect on cell activities, acting as a switch between certain functions, and also regulating the cell microenvironment (Zanzoni, Ribeiro \& Brun, 2019). Moonlighting proteins exist in various organisms, and they undertake many vital functions such as regulation, transcription, and catalysis (Irving et al., 2012; Irving, Cahill \& Chris, 2018; Świeżawska et al., 2018). These potential functions save costs and increase efficiency by producing fewer proteins from compressed genomes and completing more functions (Luís et al., 2017). It is not easy for moonlighting proteins to be discovered by biological experiments, because certain functions of some proteins are prominent or particularly important that other potential functions are easily overlooked. Therefore, effective methods are needed to summarize or identify such proteins.

Herein, we briefly reviewed the moonlighting proteins databases and some methods for identifying moonlighting proteins. In 2014, Jeffery's laboratory constructed a database MoonProt, which provides a searchable, web-based database of known moonlighting proteins, containing more than 200 experimentally verified moonlighting proteins (Mathew et al., 2015). Until 2018, the number of proteins in the MoonProt 2.0 database increased to 370, and dozens of protein annotations with additional functions or updated information were modified (Chen et al., 2018). In the same year, Spanish researchers updated the MultitaskProtDB moonlighting protein database, compiled a series of multi-functional moonlighting proteins found in the literature. The existing MultitaskProtDB-II data increased to 694 (Luís et al., 2017). It was not until 2019 that the first comprehensive plant moonlighting protein database PlantMP (Bo et al., 2019) was constructed, which has brought a lot of inspiration to our research.

Daisuke Kihara's team has developed three tools to predict moonlighting proteins based on different protein types. Initially, they developed a program to identify moonlighting proteins by clustering GO terms (Khan et al., 2014). DextMP is a tool that used the functional descriptions of the proteins to mining moonlighting proteins from literatures (Jain, Gali \& Kihara, 2018). MPFit 
73 (Khan Ishita K \& Kihara Daisuke, 2016) used multiple features from GO and multi-omics data, 74 which is a state-of-the-art non-plant specific methods.

75

76

77

78

79

80

81

82

83

84

85

86

87

88

89

90

91

92

93

94

95

96

97

98

99

100

101

102

103

104

105

106

107

108

109

Current prediction tools are mainly to identify the moonlighting proteins of animals, microorganisms and other organisms, but not constructed for plants. The plant cell structure is quite different from animal and microbial. Most of the space in plant cells is occupied by large vacuoles in the center, the only remaining space has higher requirements for regulating cell biochemical reactions such as signal ligands, molecules, and cofactors (Wong et al., 2018). The identification method of plant moonlighting protein is different from other proteins. Moreover, the experimental results on plant proteins also prove that the existing tools are not accurate enough. Due to the particularity of plant cell structure, a suitable approach is needed to discover the moonlighting protein of plants. In this context, studying a machine-learning-based plant moonlighting protein prediction tool can better serve the work of plant science and proteomics. In this article we proposed a new moonlighting protein prediction tool, IdentPMP (Identification of Plant Moonlighting Proteins), which used a benchmark data set from multiple different plant species to train the model. We extracted multiple protein feature classes, and selected the features that perform best to construct the prediction model. We expect that the plant proteins could be classified more accurately when introduced into the newly constructed IdentPMP tool.

\section{Materials \& Methods}

The entire construction process of IdentPMP includes data preparation, feature engineering, construction and prediction models evaluation. The detailed experimental procedure is described next.

\section{Data preparation}

PlantMP (Bo et al., 2019) is a relatively comprehensive moonlighting proteins Database of a plant. It contains 147 proteins involving 13 plant species, with Arabidopsis being the most abundant. The majority of data in PlantMP is extracted from PubMed articles (Bo et al., 2019). Besides, to expand the data set, we manually screened some moonlighting protein databases. 40 plant moonlighting proteins were found, including five in the MoonProt 2.0 database (Chen et al., 2018) and 35 in MultitaskProtDB-II (Luís et al., 2017). After removing some proteins that are not recognized by UniProt (Apweiler, 2004) and are unable to obtain sequences, the remaining 152 were used as positive samples in the data set. Among them, 40 proteins from MoonProt 2.0 and MultitaskProtDB-II were used as positive data in the independent test set, and 112 proteins in PlantMP were used as positive data in the training set.

In order to obtain sufficient negative samples for training models, some single-function plant proteins were selected through the following steps. First, we collected 60,000 proteins from eight species of Arabidopsis, Hordeum, Pisum sativum, Oryza sativa, Nicotiana, Pea, Moss and Zea mays on UniProt, to avoid the redundancy of a single species. Second, we used the DAVID tool 
110 (Jiao et al., 2012) to get GO terms annotation of 60000 plant proteins, and selected those proteins

111 with at least $3 \mathrm{GO}$ terms in the Biological Process (BP). Next, we used the GOSemSim package 112 (Wang, 2010) to calculate the semantic similarity of several GO terms for each protein. If the 113 semantic similarity score of several GO terms for one protein is between 0.6 and 1, indicating 114 that the GO terms of this protein have similar meanings, and we regarded it as a plant non115 moonlighting protein. Through this process, 306 negative samples (188 Arabidopsis, 58 Oryza 116 sativa, 43 Zea mays, 4 Hordeum vulgare, 9 Nicotiana, 2 Pea, 2 Moss) were selected. For each 117 species, we selected two-thirds of the data as the training data, and round-down (round-up) the 118 indivisible data to the nearest integer.

119 Next, to reduce effect of redundant sequences in the data set, we apply a technique to decrease the redundant sequences by the Cd-hit (Li \& Godzik, 2006). Cd-hit is a practical tool for clustering

121

122

123

124

125

126

127

128

129

130

131

132

133

134

135

136

137

138

139

140

141

142

143

144

145

146 biological sequences to reduce sequence redundancy, and increase the significance of other sequence. We use the command 'cd-hit' to process the training set with a threshold of 0.7. And use the command 'cd-hit-2d' to decrease the sequence redundancy between the test set and the training set. The result shows that there are 103 and 35 for the training and test set in positive samples, respectively. And in negative samples, there are 155 and 90 for the training and test set, respectively. These data constitute the benchmark data set, and the description of the data set sources can be seen in Fig. 1A.

\section{Feature engineering}

To construct an initial feature pool of plant proteins, obtaining the protein sequence in fasta format on UniProt is preliminary work. Next, we used a tool for protein feature extraction, iLearn is an ensemble platform for feature engineering analysis modeling of DNA, RNA and protein sequence data (Chen Zhen et al., 2019). We extracted 16 of feature classes using prepared sequences by iLearn, feature refers to a feature value used to train the model, feature class refers to a group of some features. The 16 feature classes including TPC (Tri-Peptide Composition), CKSAAP (Composition of k-spaced Amino Acid Pairs), CKSAAGP (Composition of kSpaced Amino Acid Group Pairs), KSCTriad (k-Spaced Conjoint Triad), DDE (Dipeptide Deviation from Expected Mean), CTDD (Distribution), Moran (Moran correlation), GTPC (Grouped Tri-Peptide Composition), Geary (Geary correlation), NMBroto (Normalized Moreau-Broto Autocorrelation), QSOrder (Quasi-sequence-order), CTDC (Composition), CTDT (Transition), PAAC (Pseudo-Amino Acid Composition), SOCNumber (Sequence-Order-Coupling Number), APAAC (Amphiphilic PseudoAmino Acid Composition).

TPC is the feature class with the most features. The TPC includes 8000 features, defined as:

$$
f(r, s, t)=\frac{N_{r s t}}{N-2}, \quad r, s, t \in\{A, C, D, \ldots, Y\}
$$

where $\mathrm{N}_{\text {rst }}$ is the number of tripeptides represented by amino acid types $\mathrm{r}$, s and $\mathrm{t}$ (Bhasin \& Raghava, 2004). Tripeptides are composed of three amino acids linked by a peptide bond, its properties and functions are determined by the presence of amino acids and the order in which 
147 they appear. The brief introduction of other feature classes can be seen in Supplementary Table 148 S1.

149 Subsequently, each type of feature data in feature pool is processed separately. Here, we

150

151

152

153

154

155

156

157

158

159

160

161

162

163

164

165

166

167

168

169

170

171

172

173

174

175

176

177

178

179

180

181

182

183 briefly describe the three major steps in the following (Fig. 1B). (1) For the first step, a wellknown feature selection technique, Information Gain (IG), was adopted. IG measures the amount of information in bits with respect to the class prediction (Chen et al., 2010; Chyh-Ming et al., 2016). The predictive accuracy of the classifier solely depends on the information gained during the training process. We use an information entropy greater than 0.05 as a threshold to determine the number of feature selections for each feature class. There are some feature classes in which the information entropy of all features is less than 0,05 . Although these feature classes are less effective in constructing models, we did not discard it. For these feature classes, we selected the top $80 \%$ of the features with information entropy. The feature dimension extracted by all feature classes can be queried in Supplementary Table S1 (We have added experiments on feature classes with information entropy less than 0.05 . The results of feature selection $70 \%, 80 \%$, and 90\% are shown in Supplementary Table S2, Supplementary Table S3, and Supplementary Table S4). (2) Secondly, the feature class are in different orders of magnitude. In order to solve the comparability between the characteristic indexes, make the process of the optimal solution smooth, and improve the calculation accuracy, this experiment used the minimax normalization method (Shalabi, Shaaban \& Kasasbeh, 2006). So that the indexes are in the same order of magnitude, which is convenient for comprehensive comparison. (3) The third step of data processing is dimension reduction. The method we chosen is PCA (Principal Component Analysis), which is used to decompose a multivariate dataset in a set of successive orthogonal components that explain a maximum amount of the variance, and each feature class is reduced to 10 dimensions (F.R.S, 1901).

After dimension reduction in the previous step, the 16 classifiers were constructed using each of the 16 feature classes by Support Vector Machine (SVM) (Furey et al., 2000). The selection of kernel and parameters of SVM has an important effect on the performance of classifier, and we use grid-search algorithm to choose the optimal parameters of SVM. Then the performances of classifiers have been evaluated by 5 -fold cross-validation, all training sets are randomly divided into five equally sized subsets. The cross-validation process is performed five times, and in each validation, a subset is selected as the test set and the remaining four as the training set. After constructing the 16 classifiers, we analyze the performance of each classifier and rank it. The feature classes used by the best performing classifier will be used in our prediction tool.

\section{Construction and prediction models evaluation}

It is important to choose a suitable classification prediction algorithm. For this purpose, we used four algorithms, Extreme Gradient Boosting (XGBoost), Support Vector Machine (SVM), Random Forest (RF), Decision Tree (DT) and K-Nearest Neighbour (KNN) to build the 
184 prediction models for plant moonlighting proteins. The selection of the model algorithm is 185 shown in the flowchart Fig. 1C.

186 SVM is a powerful machine learning algorithm for binary classification (Furey et al., 2000). It 187 aims to accurately classify samples by generating optimal hyperplanes based on the feature 188 dimensionality of the training data (Vapnik \& V., 1999). RF is well-established and widely 189 employed algorithm, which has been applied for many bioinformatics applications (Jia et al., 190 2016). It is essentially an ensemble of a number of decision trees, built on $\mathrm{N}$ random subsets of 191 the training data, and the average prediction performance is usually reported in order to avoid 192 over-fitting (Breiman, 2001). XGBoost is an ensemble algorithm, which is scalable machine 193 learning system for tree boosting, and based on the integration of classification and regression trees(Chen \& Guestrin, 2016). DT apply a tree-shaped decision model and only contains conditional control statements, which is a common and effective classification algorithm. KNN algorithm is another commonly employed unsupervised algorithm that clusters samples by calculating their similarities (Cai et al., 2012). This method is easy to understand and implement.

Among the existing related tools, MPfit is a suitable choice for comparison with our prediction tool. We compared the performance of IdentPMP and MPfit on the independent test set. To compare different machine learning methods for constructing prediction models from the training set model, we used 6 commonly used metrics, including AUPRC (area under the precision-recall curve), AUC (area under the receiver operating characteristic curve), MCC (Mathews correlation coefficient), F1-score, sensitivity and specificity. Among them, sensitivity is the true positive rate, which refers to the proportion of samples that are actually positive, specificity is the true negative rate, which refers to the proportion of samples that are actually negative. AUPRC, AUC are commonly used comprehensive evaluation metrics. IdentPMP is aim to predict plant moonlighting proteins, which are positive samples. Compared with AUC, AUPRC can better evaluate a model's ability to correctly predict and select positive samples, and is a more suitable metric for evaluating IdentPMP. AUPRC has higher requirements for positive samples in its evaluation performance, and when the prediction of positive samples is incorrect, the penalty will increase. This is more in line with our expectation to identify moonlighting protein. Therefore, we regard AUPRC as the primary metric in this experiment.

\section{Results and Discussion}

\section{Selection and analysis of feature class}

216 The 16 feature classes extracted through iLearn, was mentioned in the previous section to 217 construct classifiers, respectively using SVM algorithms. We used AUPRC as the primary 218 metric, it can take a relatively comprehensive evaluation of the model. AUC is also regarded as 219 an essential metrics. They do not need to set any specific threshold when evaluating model performance. Besides, the threshold-based metrics are calculated as minor criteria, i.e., Sensitivity, Specificity, MCC, F1-score. From Supplementary Table S3, we can see that TPC 
222 performs much better than other feature classes. Anishetty et al. (Anishetty, Pennathur \& 223 Anishetty, 2002) demonstrated that the tripeptide might be used to predict plausible structures for 224 oligopeptides and de novo protein design. Tripeptide motifs represent potentially crucial for the 225 design of small-molecule biological modulators.

226

227

228

229

230

231

232

233

234

235

236

237

238

239

240

241

242

243

244

245

246

247

248

249

250

251

252

253

254

255

256

257

258

We also integrated TPC with other feature classes to construct models, but the results were not as good as using TPC alone. That shows that integrating these feature classes do not necessarily improve performance. The possible reason is that the feature classes extracted by iLearn are sequence-based, and TPC already contains useful features related to the sequence, including redundant information with other features. Based on the above discussion, we adopted TPC to predict plant moonlighting protein and then built the predictive model in the following study.

\section{Comparison of different classification algorithms}

There are various differences in each classification algorithms. Using different classification algorithms to build models will affect the performance of prediction tools. We compared five commonly used algorithms (SVM, RF, XGBoost, DT and KNN) to analyze the impact of different algorithms on performance. We used the TPC feature class to train the classifier with five algorithms, respectively, and used the adaptive optimization method of grid search to optimize the learning model.

The 5-fold cross validation and training set results of five classifiers were shown in Table 1 . As we can see from the table, the XGBoost algorithm has the best performance on AUPRC, AUC, Sensitivity, MCC and F1-score. To show the performance of these metrics more clearly, we plotted AUPRC and AUC curves of different classifiers in Fig. 2. Then we can observe that AUPRC (AUPRC $=0.85$ ) of XGBoost is better than other algorithms, the AUC results of XGBoost and SVM are similar at 0.87 . In conclusion, XGBoost provides stronger identification capability than the other algorithms and is more appropriate for handling with the experiment of distinguishing plant moonlighting proteins from non-moonlighting proteins. Moreover, the results of the five algorithms on the independent test set are shown in Supplementary Table S5, it can be seen that XGBoost performs better in the comprehensive metric with 0.5 as the threshold. We checked the predicted result and analyzed some of the proteins that were predicted to be positive samples. The protein which UniProt ID is Q9ZVR7 is predicted as a moonlighting protein by IdentPMP. A recent article have analyzed this protein and confirmed that it is a moonlighting protein(Turek \& Irving, 2021). Q38970, which is predicted to be a moonlighting protein, and its different functions have also been confirmed in two papers (Lally et al., 2019; Gross et al., 2019).

In summary, the XGBoost algorithm outperformed other algorithms on most metrics. The AUPRC value on the training set is also the highest, which can better evaluate the model's ability to correctly select positive samples. This is in line with our purpose of constructing IdentPMP. 


\section{3. IdentPMP outperforms other method}

260 From the above steps, we finally chose XGBoost algorithm to construct IdentPMP. For 261 performance evaluation, we compared the performance of IdentPMP and MPFit (Khan Ishita K 262 \& Kihara Daisuke, 2016)on the independent test set. MPFit is a calculation tool constructed by 263 Khan et al. to predicting moonlighting proteins, mainly in the species of microorganisms and 264 animals. This tool uses a variety of features, including Phylo (phylogenetic profiles), genetic 265 interactions (GI), GE (gene expression profiles), DOR (disordered protein regions), NET (protein's graph properties in the PPI network), PPI network. The two feature combinations MPFit (Phylo+DOR+NET+GE+GI) and MPFit (Phylo+PPI+GE) are recommended by the author. Then we used these two combinations to perform experiments on plant proteins.(Khan, Mcgraw \& Kihara, 2017).

In order to illustrate and compare the performance of IdentPMP with MPFit, we plotted the results of independent test set in Fig. 3. As we can see, the result shows that the AUPRC and the AUC of IdentPMP is 0.43 and 0.68 , which are $19.44 \%$ ( 0.43 vs. 0.36$)$ and $13.33 \%$ (0.68 vs. 0.6) higher than others, respectively. Other evaluation metrics can be seen in Table 2, IdentPMP is significantly better than any feature combination of MPFit. The accuracy of positive and negative samples for the MPFit (Phylo+DOR+NET+GE+GI) was 54\% and 64\%, respectively. When the MPFit (Phylo+PPI+GE) is executed, all samples are predicted to be positive samples. The physiological characteristics of plant and other species are quite different, and there is no plant data in the training set of other tools, which may be the reason for the low accuracy of other tools in predicting plant protein. IdentPMP used a variety of plant data and the TPC determined by plant characteristics to make the prediction of plant proteins perform well.

In summary, the observed results suggest that IdentPMP is a better and easier to use predictor specifically designed for plants. It can also be proved that a clearly defined benchmark data set containing both positive and negative samples is needed for the research on plant moonlighting 284 proteins.

\section{Conclusions}

287

288

289

290

291

292

293

294
In this work, we constructed a benchmark data set and utilized feature class (TPC) to identify plant moonlighting protein. Then, we used sequence-based learning models to build a web-based prediction tool, IdentPMP. It is an integrated open-source tool for predicting the moonlighting proteins derived from plant species. As far as we know, the existing moonlighting protein prediction tools mainly focus on the proteins in animals and microorganisms. The IdentPMP is the first attempt to build a moonlighting protein prediction tool specific for plants. And it can be seen from the IdentPMP, and other tool's prediction results that the prediction tool proposed here has a better performance. 
295

296

297

298

299

300

301

302

303

304

305

306

307

308

309

310

311

312

313

314

315

316

317

318

319

320

321

322

323

324

325

326

327

328

329

Although IdentPMP performs well, there is still room for improvement. In future experiments, we will expand the data set to discover more plant moonlighting proteins using text mining, biological experiments and other methods. Using more advanced or powerful algorithms to build the model to improve the performance of prediction tools. At present, IdentPMP and the benchmark data set have filled the research gap of plant moonlighting protein. Its design principles and strategies can inspire bioinformatics to develop ideas for improved methods and can be applied to other research topics in moonlighting protein analysis. We hope that IdentPMP will bring some benefits to the research field of plant moonlighting proteins.

\section{References}

Akinyelu AA, Adewumi AO. 2013. Classification of Phishing Email Using Random Forest Machine Learning Technique. Journal of Applied Mathematics. DOI: 10.1155/2014/425731.

Anishetty S, Pennathur G, Anishetty R. 2002. Tripeptide analysis of protein structures. BMC Structural Biology 2:9. DOI: 10.1186/1472-6807-2-9.

Apweiler R. 2004. UniProt: The Universal Protein knowledgebase. Nucleic Acids Research 32:115D - 119. DOI: 10.1093/nar/gkh131.

Bhasin M, Raghava GPS. 2004. Classification of nuclear receptors based on amino acid composition and dipeptide composition. Journal of Biological Chemistry. DOI: 10.1074/jbc.M401932200.

Bo, Su, Zhuang, Qian, Tianshu, Li, Yuwei, Zhou, Aloysius, Wong. 2019. PlantMP: a database for moonlighting plant proteins. Database the Journal of Biological Databases \& Curation. DOI: $10.1093 /$ database/baz050.

Breiman L. 2001. Random forest. Machine Learning 45:5-32.

Cai Y, Huang T, Hu L, Shi X, Xie L, Li Y. 2012. Prediction of lysine ubiquitination with mRMR feature selection and analysis. Amino Acids 42:1387-1395. DOI: 10.1007/s00726-011-0835-0. Chen T, Guestrin C. 2016. XGBoost: A Scalable Tree Boosting System. In: Proceedings of the 22nd ACM SIGKDD International Conference on Knowledge Discovery and Data Mining. KDD '16. New York, NY, USA: Association for Computing Machinery, 785-794. DOI: $10.1145 / 2939672.2939785$.

Chen K, Jiang Y, Du L, Kurgan L. 2010. Prediction of integral membrane protein type by collocated hydrophobic amino acid pairs. Journal of Computational Chemistry 30:163-172. DOI: $10.1002 / \mathrm{jcc} .21053$.

Chen K, Kurgan LA, Ruan J. 2007. Prediction of flexible/rigid regions from protein sequences using k-spaced amino acid pairs. BMC Structural Biology 7:25. DOI: 10.1186/1472-6807-7-25. 
330 Chen C, Shadi Z, Liu H, Wang W, Constance J. 2018. MoonProt 2.0: an expansion and update of 331 the moonlighting proteins database. Nuclc Acids Research:D640. DOI: 10.1093/nar/gkx1043.

332 Chen Zhen, Zhao Pei, Li Fuyi, Marquez-Lago Tatiana T, Leier André, Revote Jerico, Zhu Yan, 333 Powell David R, Akutsu Tatsuya, Webb Geoffrey I. 2019. iLearn: an integrated platform and 334 meta-learner for feature engineering, machine-learning analysis and modeling of DNA, RNA and 335 protein sequence data. Briefings in Bioinformatics. DOI: 10.1093/bib/bbz041.

336 Chyh-Ming, Lai, Wei-Chang, Yeh, Chung-Yi, Chang. 2016. Gene selection using information 337 gain and improved simplified swarm optimization. Neurocomputing. DOI:

338 10.1016/j.neucom.2016.08.089.

339 F.R.S KP. 1901. LIII. On lines and planes of closest fit to systems of points in space. The

340 London, Edinburgh, and Dublin Philosophical Magazine and Journal of Science 2:559-572.

341 DOI: 10.1080/14786440109462720.

342 Furey TS, Cristianini N, Duffy N, Bednarski DW, Schummer M, Haussler D. 2000. Support

343 vector machine classification and validation of cancer tissue samples using microarray

344 expression data. Bioinformatics 16:906-14. DOI: 10.1093/bioinformatics/16.10.906.

345 Gross AS, Zimmermann A, Pendl T, Eisenberg T. 2019. Acetyl-CoA carboxylase 1-dependent 346 lipogenesis promotes autophagy downstream of AMPK. The Journal of Biological Chemistry

347 294:12020-12039. DOI: 10.1074/jbc.RA118.007020.

348 Irving HR, Cahill DM, Chris G. 2018. Moonlighting Proteins and Their Role in the Control of

349 Signaling Microenvironments, as Exemplified by cGMP and Phytosulfokine Receptor 1

350 (PSKR1). Frontiers in Plant Ence 9:415. DOI: 10.3389/fpls.2018.00415.

351 Irving HR, Kwezi L, Wheeler J, Gehring C. 2012. Moonlighting kinases with guanylate cyclase 352 activity can tune regulatory signal networks. DOI: 10.4161/psb.18891.

353 Jain A, Gali H, Kihara D. 2018. Identification of Moonlighting Proteins in Genomes Using Text 354 Mining Techniques. PROTEOMICS. DOI: 10.1002/pmic.201800083.

355 Jeffery CJ. 1999. Moonlighting Proteins. Trends in Biochemical Sciences 24:8-11. DOI:

356 10.1016/S0968-0004(98)01335-8.

357 Jia J, Liu Z, Xiao X, Liu B, Chou KC. 2016. pSuc-Lys: Predict lysine succinylation sites in 358 proteins with PseAAC and ensemble random forest approach. Journal of Theoretical 359 Biology:223-230. DOI: 10.1016/j.jtbi.2016.01.020.

360 Jiao X, Sherman BT, Da WH, Stephens R, Lempicki RA. 2012. DAVID-WS: A stateful web 361 service to facilitate gene/protein list analysis. Bioinformatics 28:1805-1806. DOI:

362 10.1093/bioinformatics/bts251.

363 Kawashima Shuichi, Pokarowski Piotr, Pokarowska Maria. 2008. AAindex: amino acid index 364 database, progress report 2008. Nuclc Acids Research. DOI: 10.1093/nar/gkm998. 
365 Ke Chen, Lukasz Kurgan, Mandana Rahbari. 2007. Prediction of protein crystallization using 366 collocation of amino acid pairs. Biochemical \& Biophysical Research Communications. DOI: 367 10.1016/j.bbrc.2007.02.040.

368 Khan I, Chen Y, Dong T, Hong X, Takeuchi R, Mori H, Kihara D. 2014. Genome-scale 369 identification and characterization of moonlighting proteins. Biology Direct 9:30. DOI: 370 10.1186/s13062-014-0030-9.

371 Khan Ishita K, Kihara Daisuke. 2016. Genome-scale prediction of moonlighting proteins using 372 diverse protein association information. Bioinformatics. DOI: 10.1093/bioinformatics/btw166.

373 Khan I, Mcgraw J, Kihara D. 2017. MPFit: Computational Tool for Predicting Moonlighting 374 Proteins. Methods Mol Biol:45-57. DOI: 10.1007/978-1-4939-7015-5_5.

375 Lally JSV, Ghoshal S, Fuchs BC. 2019. Inhibition of Acetyl-CoA Carboxylase by 376 Phosphorylation or the Inhibitor ND-654 Suppresses Lipogenesis and Hepatocellular Carcinoma. 377 Cell Metabolism 29:174-182.e5. DOI: 10.1016/j.cmet.2018.08.020

378 Li W, Godzik A. 2006. Cd-hit: a fast program for clustering and comparing large sets of protein 379 or nucleotide sequences. Bioinformatics 22:1658-1659. DOI: 10.1093/bioinformatics/btl158.

380 Lin H, Ding C, Yuan L-F, Chen W, Ding H, Li Z-Q, Guo F-B, Huang J, Rao N-N. 2013.

381 Predicting subchloroplast locations of proteins based on the general form of chou's pseudo 382 amino acid composition: approached from optimal tripeptide composition. International Journal 383 of Biomathematics 06:1350003. DOI: 10.1142/S1793524513500034.

384 Luís FS, Sergio H, Alejandra C, Severi MA, Gabriela F, Josepantoni PP, Jaume P, Òscar P, 385 Ángel MV, Isaac A. 2017. MultitaskProtDB-II: an update of a database of 386 multitasking/moonlighting proteins. Nuclc Acids Research:D1. DOI: 10.1093/nar/gkx1066.

387 Mathew M, Chen C, Vaishak A, Liu H, Tanu M, Grant Z, Shadi Z, Bansi P, Jagravi T, Jeffery 388 CJ. 2015. MoonProt: a database for proteins that are known to moonlight. Nuclc Acids 389 Research:534a-534a. DOI: 10.1016/j.bpj.2014.11.2928.

390 Mitchell TM. 1997. Machine learning (mcgraw-hill. DOI: http://dx.doi.org/.

391 Shalabi LA, Shaaban Z, Kasasbeh B. 2006. Data Mining: A Preprocessing Engine. DOI: $39210.3844 /$ jcssp.2006.735.739.

393 Sokal RR, Thomson BA. 2010. Population structure inferred by local spatial autocorrelation: An 394 example from an Amerindian tribal population. American Journal of Physical Anthropology 395 129:121-131. DOI: 10.1002/ajpa.20250.

396 Świeżawska B, Duszyn M, Jaworski K, Szmidt-Jaworska A. 2018. Downstream Targets of 397 Cyclic Nucleotides in Plants. Frontiers in Plant Science 9. DOI: 10.3389/fpls.2018.01428.

398 Turek I, Irving H. 2021. Moonlighting Proteins Shine New Light on Molecular Signaling Niches. 399 International Journal of Molecular Sciences 22:1367. DOI: 10.3390/ijms22031367. 
400 Vapnik, V. N. 1999. An overview of statistical learning theory. IEEE Trans Neural Netw 401 10:988-999. DOI: 10.1109/72.788640.

402 Vijayakumar, Saravanan, Namasivayam, Gautham. 2015. Harnessing Computational Biology for 403 Exact Linear B-Cell Epitope Prediction: A Novel Amino Acid Composition-Based Feature 404 Descriptor. Omics A Journal of Integrative Biology. DOI: 10.1089/omi.2015.0095.

405 Wang S. 2010. GOSemSim: an R package for measuring semantic similarity among GO terms 406 and gene products. Bioinformatics 26:976-978. DOI: 10.1093/bioinformatics/btq064.

407 Wong A, Tian X, Gehring C, Marondedze C. 2018. Discovery of Novel Functional Centers With 408 Rationally Designed Amino Acid Motifs. Computational and Structural Biotechnology Journal 409 16:70-76. DOI: 10.1016/j.csbj.2018.02.007.

410 Zanzoni A, Ribeiro DM, Brun C. 2019. Understanding protein multifunctionality: from short 411 linear motifs to cellular functions. Cellular and Molecular Life Sciences 76:4407-4412. DOI: 412 10.1007/s00018-019-03273-4.

413

414 
Figure 1

The flowchart of IdentPMP development.

(A) Data preparation. The composition and source of training data set and independent test data set. (B) Feature engineering. We used iLearn to generate feature classes, perform preprocessing, and use each feature class to construct a classifier to select the best feature. (C) Model training. Five algorithms are used, including Random Forest (RF), Support Vector Machine (SVM), Extreme Gradient Boosting (XGBoost), Decision Tree (DT) and K-Nearest Neighbour (KNN). 


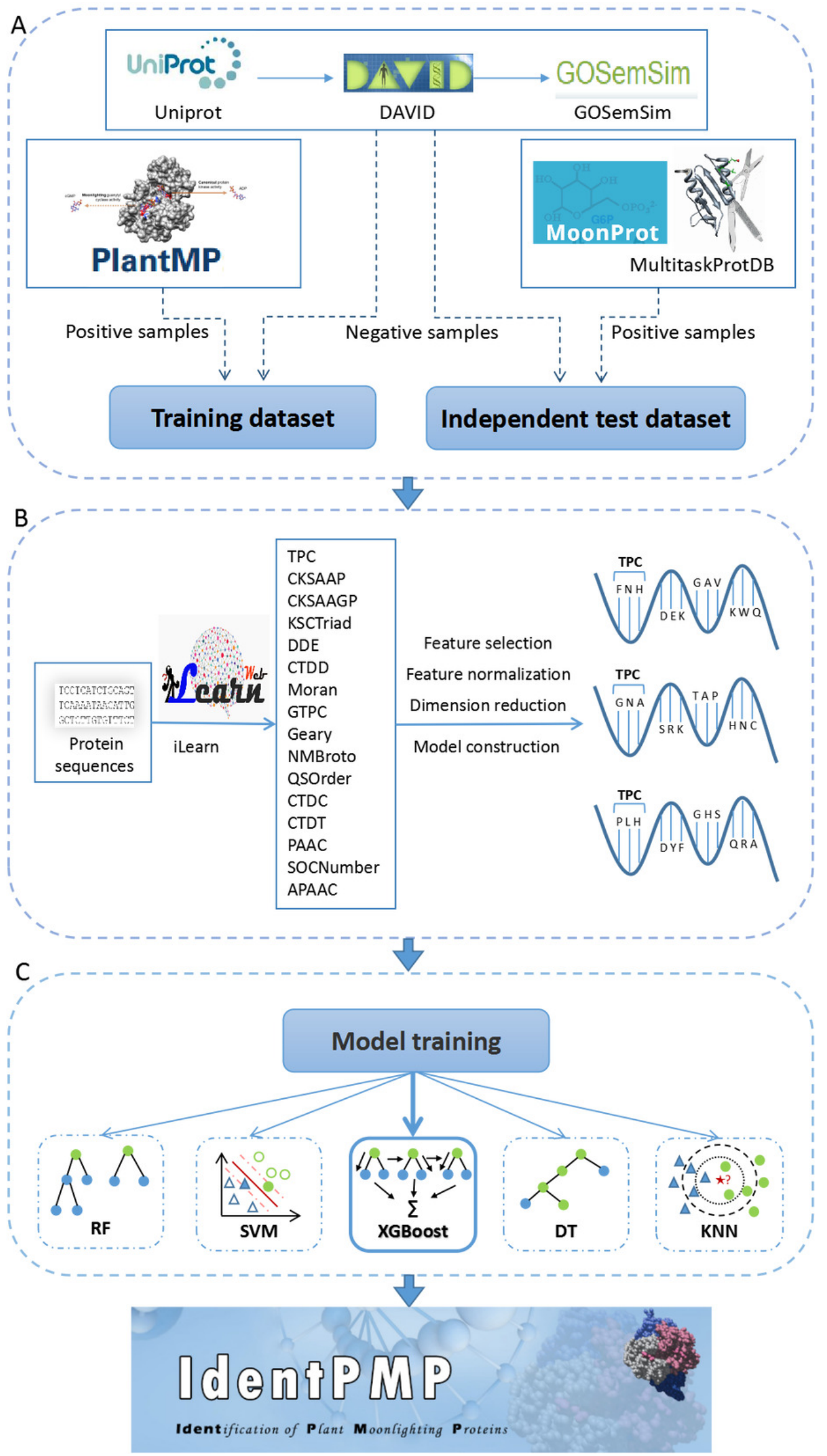


Figure 2

Performance comparison of the five algorithms on the training set.

(A) AUPRC curves of the five algorithms. (B) AUC curves of the five algorithms.
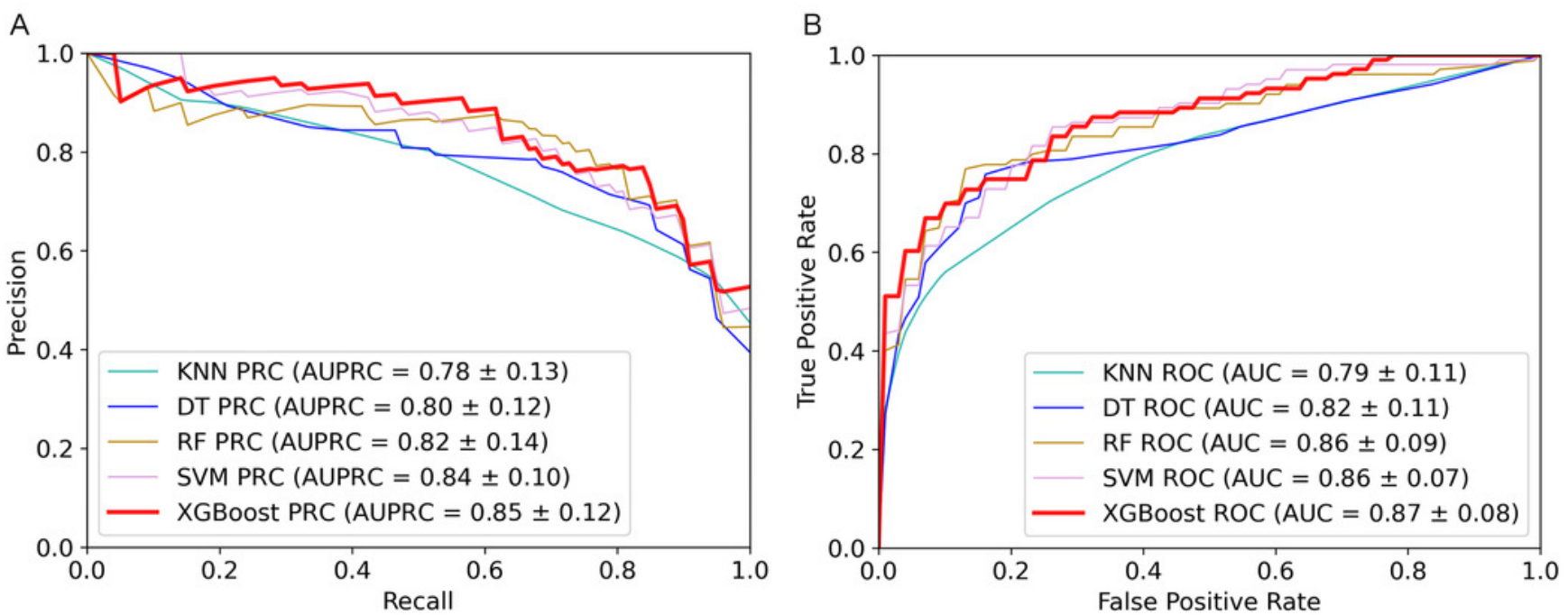
Figure 3

Performance comparison of IdentPMP and MPFit on the independent test set.

(A) AUPRC curves of the IdentPMP and MPFit (Phylo+DOR+NET+GE+GI). (B) AUC curves of the IdentPMP and MPFit (Phylo+DOR+NET+GE+Gl)
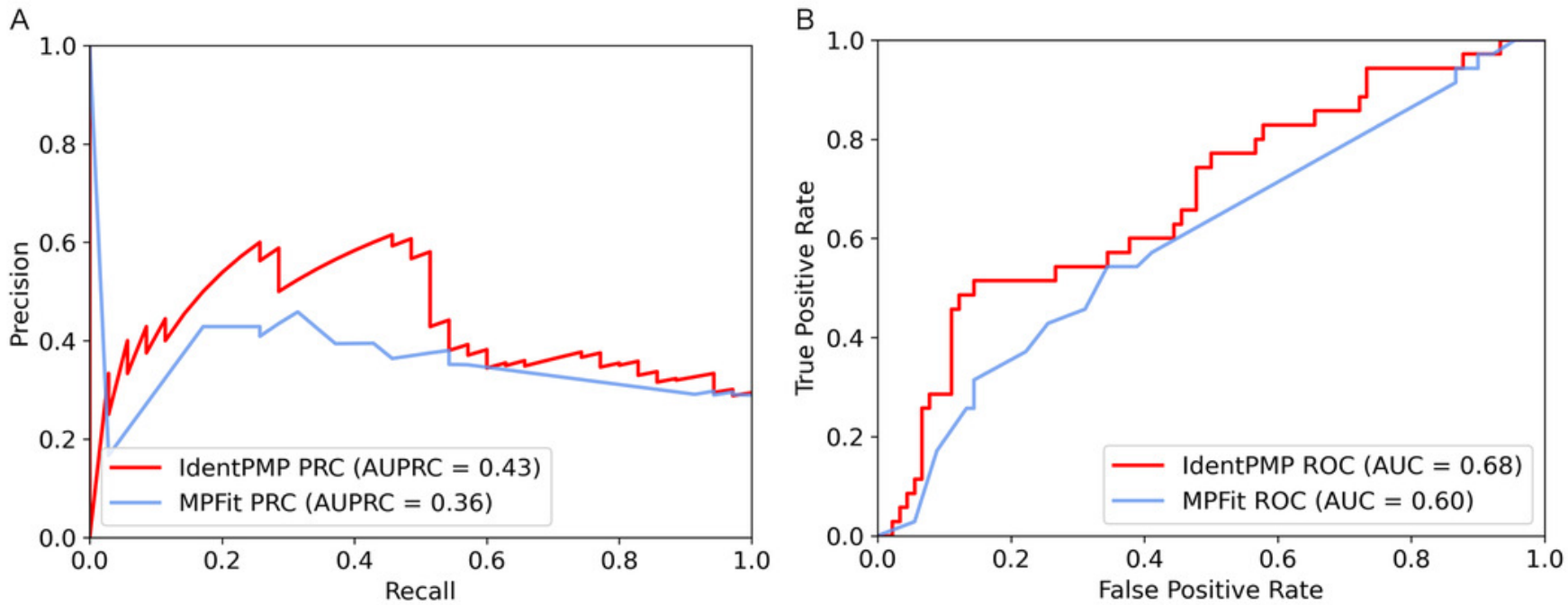


\section{Table $\mathbf{1}$ (on next page)}

The performance of five algorithms on the training set.

AUPRC, area under the precision-recall curve. AUC. area under the receiver operating characteristic curve. AUPRC is the main metric. Sen, sensitivity. Spe, specificity. MCC, Matthews correlation coefficient. F1, F1-score. The selected algorithm and The maximum values in each metric are marked in bold. 
1

\begin{tabular}{lcccccc}
\hline Method & AUPRC & AUC & Sen & Spe & MCC & F1 \\
\hline XGBoost & $\mathbf{0 . 8 5}$ & $\mathbf{0 . 8 7}$ & $\mathbf{0 . 6 8}$ & 0.90 & $\mathbf{0 . 6 2}$ & $\mathbf{0 . 7 4}$ \\
SVM & 0.84 & 0.86 & 0.68 & 0.85 & 0.55 & 0.70 \\
RF & 0.82 & 0.86 & 0.70 & 0.85 & 0.56 & 0.72 \\
DT & 0.80 & 0.82 & 0.68 & 0.84 & 0.53 & 0.70 \\
KNN & 0.78 & 0.79 & 0.53 & $\mathbf{0 . 9 4}$ & 0.51 & 0.62 \\
\hline
\end{tabular}

2 


\section{Table 2 (on next page)}

The detailed values of the results of IdentPMP and MPFit on the independent test set.

AUPRC, area under the precision-recall curve. AUC, area under the receiver operating characteristic curve. Sen, sensitivity. Spe, specificity. MCC, Matthews correlation coefficient. F1, F1-score. The maximum values in each metric are marked in bold. 


\begin{tabular}{lcccccc}
\hline Method & AUPRC & AUC & Sen & Spe & MCC & F1 \\
\hline IdentPMP & 0.43 & $\mathbf{0 . 6 8}$ & 0.46 & $\mathbf{0 . 8 9}$ & $\mathbf{0 . 3 7}$ & $\mathbf{0 . 5 2}$ \\
MPFit(Phylo+GE+GI+DOR+NET) & 0.36 & 0.60 & 0.54 & 0.64 & 0.17 & 0.44 \\
MPFit(PPI+Phylo+GE) & $\mathbf{0 . 6 4}$ & 0.50 & $\mathbf{1 . 0 0}$ & 0.00 & 0.00 & 0.43 \\
\hline
\end{tabular}

1 